Man and Nature

L'homme et la nature

\title{
Le masque et l'éventail
}

\section{Pierre Gobin}

Volume 7, 1988

URI : https://id.erudit.org/iderudit/1011926ar

DOI : https://doi.org/10.7202/1011926ar

Aller au sommaire du numéro

Éditeur(s)

Canadian Society for Eighteenth-Century Studies / Société canadienne d'étude du dix-huitième siècle

ISSN

0824-3298 (imprimé)

1927-8810 (numérique)

Découvrir la revue

Citer cet article

Gobin, P. (1988). Le masque et l'éventail. Man and Nature / L'homme et la nature, 7, 53-69. https://doi.org/10.7202/1011926ar

Copyright $@$ Canadian Society for Eighteenth-Century Studies / Société canadienne d'étude du dix-huitième siècle, 1988
Ce document est protégé par la loi sur le droit d'auteur. L'utilisation des services d'Érudit (y compris la reproduction) est assujettie à sa politique d'utilisation que vous pouvez consulter en ligne.

https://apropos.erudit.org/fr/usagers/politique-dutilisation/
Cet article est diffusé et préservé par Érudit.

Érudit est un consortium interuniversitaire sans but lucratif composé de l’Université de Montréal, l'Université Laval et l'Université du Québec à Montréal. Il a pour mission la promotion et la valorisation de la recherche. https://www.erudit.org/fr/ 


\section{Le masque et l'éventail}

Au commencement est la contrainte. Alors même qu'elles se laïcisent, du Discours sur l'origine de l'inégalité à la Phénoménologie de l'Esprit, les visions globales de l'histoire humaine transposent le récit du péché originel, de la chute, du châtiment. Pourtant, au siècle des Lumières, il ne s'agit plus (ou presque plus) de s'en remettre à un Rédempteur pour rendre aux humains leur dignité, il ne s'agit pas encore (ou presque pas) d'appeler à suivre l'exemple d'un Révolté prométhéen. ${ }^{1}$ Ni la résignation invoquant la Grâce (et la Révélation) ni le défi (conduisant à la Révolution) ne constituent des réponses acceptables aux esprits qui tentent de fonder le bonheur ici-bas. Les plus optimistes osent croire que 'l'esprit du siècle' est 'celui de la liberté.'2 Mais tous savent que les appareils répressifs humains ne cessent de reprendre - sous des formes abusives et dégradées, bien qu'elles invoquent le Droit - les gestes punitifs de la divinité offensée. Dans ces conditions si l'on refuse de se soumettre, il faut être prudent; mais le conformisme 'sage' ou la démarche de l'acteur pris de trac qui s'avance masqué ('larvatus prodeo') proposés par Descartes non plus que le divertissement pascalien, ressource des libertins d'autrefois, ne sont maintenant de mise. C'est qu'on est prêt à agir sur le monde plutôt que sur soi.

Parfois on procède de façon somme toute grossière: c'est le 'frapper et cacher sa main' préconisé par Voltaire, tactique frondeuse et sournoise mais simpliste du gamin tireur de sonnettes. Souvent toutefois on élabore des stratégies plus subtiles. Voltaire multiplie les intervenants, soit en faisant donner les 'frères,' soit en inventant tout un escadron volant de personae qui harcèlent de leurs facéties les lourdes colonnes des appareils d'état. Diderot perfectionne le méthode de Bayle et lance ses censeurs dans un labyrinthe de citations et de renvois ( $L^{\prime} E n$ cyclopédie) ou passe sa marchandise sous le pavillon d'autrui (les abbés sont particulièrement utiles, de Prades à Raynal en passant par Yvon et Morellet). Les mêmes et d'autres jouent au furet entre la fiction (peutêtre fondée dans un réel à clefs multiples) et l'aveu (peut-être truqué). Les privilèges traditionnels des fabulistes et des dramaturges (cautions mimétiques/leurres ou effets de 'trompe l'esprit'/justifications morales) sont bien sûr invoqués; mais les cautions s'articulent avec plus de souplesse (éditeurs 'à tiroirs' des 'mémoires' prétendus; correspondances 
polylogiques des romans par lettres), tandis que les leurres se font plus incertains 'et d'autant plus trompeurs qu'ils disent parfois vrai' (glissement du personnage au type - et vice versa - dans les comédies; jeux de 'miroirs vides'; ${ }^{3}$ relativisation du temps, de l'espace, de l'identité des personnages; 'parodisation' des utopies, et 'utopisation' des parodies selon des modalités dont Swift et Marivaux semblent avoir donné les premiers exemples réussis; mystifications et persiflages 'sérieux'; pertinence de l'impertinence, ${ }^{4}$ et j'en passe). Quant aux justifications du type 'castigo ridendo mores,' elles seront rendues plus difficiles après la roide attaque de la Lettre à d'Alembert. On tendra dès lors à les subsumer au sein d'un vaste projet progressiste, rappelé sur le mode sentimental (Marmontel, La Harpe), ou ironique (Chamfort), vertueusement indigné (Mercier, Beaumarchais) ou stoïque (Diderot, dans $L^{\prime} E s-$ sai sur les règnes de Claude et de Néron) à moins qu'on ne les élude en les relativisant (Jacques le Fataliste) ou, à la limite qu'on ne les récuse par un 'suprême effort' (Sade) ou qu'on ne les inverse radicalement (Joseph de Maistre).

Parmi les stratégies mises au point pour déjouer la censure de l'appareil (et toutes sortes d'autres censures moins nettement perçues) figure tout un groupe, particulièrement efficace, mais curieux de par son cheminement. Ceux qui ont recours à ces stratégies passent en quelque sorte par 'l'excentrique' (comme dirait Benrekassa) pour atteindre les problèmes centraux: choix de sites exotiques (anodins parce qu'ils dépaysent), fantaisistes (du conte de fées on peut ainsi passer au conte philosophique), ou utopiques, de fables frivoles ou licencieuses (les 'fredaines' érotiques 'couvrent' le libertinage idéologique), de personnages usés par les conventions (Arlequin) ou les mythes, ou encore jugés 'sans conséquence' comme Rameau le Neveu, du temps où il était parasite. Donner la parole à ceux qui ne sauraient parler constitue en quelque sorte un leurre qui annule toute caution mimétique et crée une zone où les pouvoirs de l'appareil sont comme neutralisés (dans le Neveu de Rameau, il reprennent leur efficace dès que le bouffon manifeste son 'sens commun'). La critique même semble désarmée là où la gratuité règne, dans le lieu du badinage; les 'sociétés badines' (recensées par Arthur Dinaux), les académies et 'corps' plus ou moins fantaisistes, les salons mêmes, offrent des ressources que n'ont pas les académies véritables où siègent les 'hommes graves' tels que le Président Montesquieu. ${ }^{5}$

$C^{\prime}$ est là je crois que les femmes jouent un rôle particulièrement important, mais qui ne va pas sans ambiguités supplémentaires. Tout $\mathrm{d}^{\prime}$ abord, en assurant une caution plus solidement fondée ( $\mathrm{s}^{\prime} \mathrm{il}$ est presque impensable qu'un 'vrai' Chinois, un 'vrai' Turc ait pu s'adresser sans truchement aux lecteurs français ('comment peut-on être Persan?') 
ou qu'un individu réellement marginal s'exprime autrement que par la fiction, picaresque, grivoise ou burlesque (encore qu'il y ait lieu de reconsidérer la notion de bohème depuis les recherches de Darnton), il n'est pas rare qu'une honnête femme prenne vraiment la plume), la présence d'une narratrice ou d'une émettrice ostensibles décale le leurre. Ensuite à l'intérêt s'ajoute la séduction (qu'on se souvienne de l'admirable cri du coeur de Chérubin: 'Une fille, une femme. Ah que ces noms sont doux! qu'ils sont intéressants!') qui induit chez le lecteur ou récepteur potentiel toutes sortes d'effets de charme (bienveillance, attraction, vieilles méfiances théologiques), chez la lectrice/réceptrice une sympathie plus forte relançant le vieux 'tourniquet' qui fait passer de 'l'objet' (qui s'intériorise) au sujet (qui se projette) dans un étonnement sans terme. Le succès de la mystification à laquelle s'est livré l'obscur provincial Desforges-Mallard, qui sous les traits de la jeune poétesse Mlle Malcrais de la Vigne a pu 'faire marcher' le rusé Voltaire lui-même - à la grande joie de Piron ${ }^{6}$ - de même que la persistante 'identification féminine' du marivaudage tiennent largement à cette sympathie et à ce charme qui mériteraient une étude poussée. Je dirais que dans ces cas les masques étant peu marqués (par opposition avec ceux des exotiques ou des picaros, qui, d'emblée dépaysants, attirent l'attention sur le leurre) peuvent demeurer inaperçus tout au long de l'expérience de réception. Ce n'est qu'au XXe siècle que l'on a pu enfin établir avec certitude que les Lettres de la Religieuse Portugaise étaient apocryphes et dûes à la plume d'un homme; ${ }^{7}$ et l'on peut lire les Lettres de la Marquise de xxx sans songer que c'est Duclos leur auteur. L'expérience dramatique du lever du masque si importante chez les Élizabéthains (jouant en travesti), les dramaturges espagnols du siglo de oro (expression matérielle du disengaño), et même chez Molière ou Marivaux n'est donc pas pertinente. ${ }^{8}$ En revanche on peut jouer avec un autre 'écran,' mais cette fois un écran partiel et mobile: je veux parler de l'éventail, accessoire mondain familier, dont l'usage pratique a été secondarisé, mais dont les figures se sont développées, codées (à travers la manière de le tenir, de le déployer, de le placer, etc.); sa 'géométrie variable' permet à 'l'utilisatrice' de se révéler tout entière, et derechef de se cacher, ou encore de mettre en évidence tel détail de sa physionomie, un peu à la manière des 'mouches' de taffetas noir qui accentuent les 'beautés'; mais s'il modifie $l^{\prime \prime}$ objet' féminin en fonction des initiatives du 'sujet' lui-même, il autorise aussi le sujet à concentrer son regard.

C'est donc un instrument de la pudeur active, et comme tel il constitue une métaphore qui combine la sincérité avec ses corrélats sémiotiques, ${ }^{9}$ et permet d'approfondir une problématique du secret où caution, leurre et justification s'inscrivent dans un ensemble de mouvements 
diversements vectorisés (tout comme les signes corporels d'affirmationopiner, hocher la tête - ou de négation - par pivotement latéral, mais de façon plus souple et plus complexe encore). Qui plus est, il offre la possibilité de 'rire sous cape,' et de se rire des pouvoirs.

La valeur métaphorique de l'éventail ne s'arrête d'ailleurs pas à une série de variations sur le sens 'propre': son sens 'figuré,' établi dans une métaphore commune (primaire, pourrait-on dire) joue sur l'idée d'un faisceau d'options à partir d'une articulation commune que l'on peut ouvrir/fermer. Il présente donc la possibilité d'une classification (et ressemble au fameux arbre encyclopédique, définissant un principe structural, ou évoque le rayonnement à partir d'un foyer de diffusion comme dans la célèbre gravure de Cochin). Il peut aussi être associé à une métaphorisation secondaire par sa ressemblance avec une main qui peut s'étendre et s'ouvrir-comme celle de la semeuse jetant libéralement les graines fécondes à la terre nourricière, articulant la culture sur la nature comme les optimistes des Lumières, champions de l'éducation libre et universelle (Diderot dans ses 'bons moments,' Condorcet) - mais qui peut rester jalousement fermée sur les trésors qu'elle contient, comme le poing qui détiendrait des vérités peut-être dangereuses dont parle Fontenelle, sceptique ou pessimiste: nous retrouvons alors le rapport à la contrainte. On le sait, l'option pessimiste rejoint l'attitude la plus misogyne, dont on connaît la tentative de justification: c'est Ève qui fut séduite la première par l'esprit du mal; c'est Pandore qui a ouvert la funeste boîte; par conséquent il faut étroitement limiter la liberté accordée aux femmes qui risquent d'en faire un si mauvais usage et de perdre tous les humains avec elles. Il me semble alors que la relation des femmes avec la clôture (qui leur est imposée par une contrainte sexiste cette fois) est peut-être une des formes les plus extrêmes de cette métaphorisation secondaire. C'est pourquoi je me propose d'examiner brièvement trois romans de la claustration féminine, écrits par des hommes, qui me semblent à travers une transposition (de la caution, du leurre, de la justification) quasi-radicale dans sa 'secondarité' poser les problèmes du pouvoir et de ses abus. ${ }^{10}$

Deux de ces romans sont teintés d'exotisme. L'affabulation 'persane,' en contrepoint avec la découverte par Usbek et Rica de l'occident (une 'tentation' qu'il faudra bien un jour comparer avec celle qu'évoque Malraux) est déjà bien étudiée. Je me bornerai donc dans les Lettres de Montesquieu à l'examen de celles qui sont censées émaner du sérail (ou plutôt de ses 'pensionnaires,' le statut des eunuques impliquant un 'échange' entre la castration/tonsure? et l'exercice d'un pouvoir délégué mais absolu ${ }^{11}$ à l'intérieur d'une étroite sphère); ces lettres 'féminines' sont peut-être les plus 'romanesques' (devrais-je dire tragiques?); elles sont à coup sûr les plus éloignées du quotidien, les plus 'épurées' (les 
plus 'secondarisées' métaphoriquement). Elles établissent aussi la clôture du roman (ou le précipitent dans une catastrophe, précédée par une atroce reconnaissance, des lettres CLVI (Roxanne), CLVII (Zachi), CLVIII (Zelis), à CLXI (Roxanne mourante) alors qu'elles l'avaient situé au début (III Zachi, IV Zéphis, VII Fatmé). À ces 'délégations de parole' je joindrai cependant quelques textes 'témoins' (tels que la lettre 'programmatique' d'Usbek (XXXIV) ou la lettre $C X$ de Rica sur les femmes occidentales et leur divertissement, et les histoires en abyme (LXVII, et surtout CXLI, 'travesti' par Rica d'un conte persan).

Par ailleurs, dans l'Histoire d'une Grecque Moderne de Prévost (1740-41), que je tiens pour une oeuvre plus 'achevée' que Manon Lescaut (même si elle est moins 'touchante') et digne de figurer à côté de la Prisonnière de Proust parmi les grandes histoires de la 'grandeur et servitude amoureuse,' je concentrerai mon attention sur les pages où le narrateur rapporte les propos de Zara/Théophé (notamment les pages 23 à 46, dans l'édition 10-18 - récit des aventures de la jeune captive); les épisodes où elle fait part de ses scrupules $(95-96 ; 102-103)$, où elle rapporte des entreprises scabreuses (143-45), où elle évoque la 'sagesse' comme bien suprême ('le "terme auquel on aspire," ' 201), où elle réduit à néant les espoirs de son protecteur par une 'cruelle protestation' (207); après le départ de Constantinople, les scènes où la jeune femme tente $\mathrm{d}$ 'apaiser la jalousie de plus en plus manifeste de son protecteur au statut ambigu (234-35); enfin, à Paris (et 'Saint Clou'), le jeu complexe des ménagements galants (257), des 'plans de réforme' où figure le couvent (260), et des intrigues feutrées de 'bienséance,' qui aboutit à la grande scène de 'justification' (268-71) - laquelle demeure d'ailleurs suspendue, pour l'incertitude du narrateur (270), par l'évanouissement de Théophé devant les 'outrages' de sa duègne accusatrice (271), enfin par un retour 'civilisé' de la clôture, de la censure, de la violence imposées par le galant homme qu'est le narrateur lui-même ('je pris avec plus d'un soupir le parti de leur imposer silence ... Vous ne me quitterez point, dis-je') qui réunit tous les personnages dans un huis-clos infernal.

Le troisième texte, la Religieuse de Diderot, s'il ne recourt pas aux leurres orientaux, s'inscrit dans une mystification très élaborée s'appuyant sur des cautions habiles, et fait intervenir une justification idéologique évidente (puisqu'il s'agit de combattre les voeux forcés) mais présentée avec beaucoup de tact (le destinataire immédiat étant le bon et pieux Marquis de Croixmare, le texte ostensiblement dû à une religieuse en butte aux vexations de l'institution gomme tout ce que l'athée Diderot pourrait imputer au dogme ou à la foi). ${ }^{12}$ Contrairement aux deux autres, il ne fragmente pas la parole 'féminine.' Dans les L.P. cette parole est distribuée entre plusieurs émettrices, mise en 
cause par les 'réfutations' directes ou indirectes des délégués ou des tenants du pouvoir, enchassée dans une série de discours préfaciels (introduction de l'homme grave' qui s'efface mais s'affirme comme 'traducteur,' réflexions du critique qui rappelle 'la chaîne qui lie' toutes les lettres et suggère une loi quasi-mathématique permettant de situer chacune: 'le désordre croît dans le sérail d'Asie à proportion de la longueur de l'absence d'Usbek'). Pour ce qui est de l'H.G.M., la parole féminine est rapportée en des points choisis d'un texte recomposé, sous forme de citation à la première personne, introduite à la troisième ('J'ai commencé à me connoître me dit-elle,' 23), ou, le plus souvent de citation 'préparée' par une relation ('elle me confessa... Il est vrai, continua-t-elle que j'ai moins combattu ce penchant que je ne le devois,' 234), le statut du texte du narrateur étant lui-même mis en cause par un exorde lui-même sujet à caution par l'aveu qui l'amorce: 'Ne me rendrai-je point suspect par l'aveu qui va faire mon Exorde? Je suis l'Amant de la belle Grecque dont j'entreprends l'Histoire. Qui me croira sincère dans le récit de mes plaisirs et de mes peines?' (3). ${ }^{13}$ Chez Diderot, Suzanne est censée tenir la plume tout au long du texte. Toutefois, après la reconnaissance par l'héroïne de ce qu'était la supérieure du couvent d'Arpajon, lorsqu'elle surprend son atroce et poignante confession (' "Mon père, je suis damnée" ... Le voile ... se déchirait...'), ${ }^{14}$

Les Mémoires de la soeur Suzanne sont interrompus; ce qui suit ne sont plus apparemment que les réclames de ce qu'elle se promettait apparemment d'employer dans le reste de son récit,

ainsi que le note 'l'éditeur' (au statut particulièrement brouillé: s'agitil de 'Madame Madin'?/du compilateur de la 'préface-annexe' (Diderot/Grimm/...)?/du 'charmant marquis' mystifié lui-même qui après la 'mort' de 'la chère demoiselle' demandait communication des 'petits' mémoires qu'elle a faits de ses différents malheurs?). Tout se passe comme si le retour dans 'le siècle' s'accompagnait d'une perception nouvelle du temps. Non seulement le texte est fragmenté, mais le passé historique est abandonné pour le présent, et même pour un impératif d'adjuration phatique ('Monsieur, hâtez-vous de me secourir') qui traduit les 'alarmes continuelles' de la fugitive. Mais la menace qui pèse sur l'héroïne n'est pas la mort, du moins dans le texte même, comme pour la Roxanne des L.P., ni même l'intimidation qui la précède dans l'H.G.M. . Certes la mort viendra clore cette histoire, mais elle n'apparait que dans le hors-texte: lorsque c'est 'Suzanne' qui est censée s'exprimer, c'est la découverte qui l'angoisse ('mes étourderies me décèleront, et je serai perdue'), et le poids de la liberté même, qui risque de l'amener à se trahir; bien qu'elle ait résisté à la tentation du suicide, 
arrêtée au bord du 'puits profond, situé au bout du jardin de la maison,' par Dieu et par 'l'entière liberté' où on la laisse, elle éprouve un étrange vertige quand il s'agit d'assumer l'absence de contrainte extérieure. C'est peut-être ce qui rend les cautions de ce texte de confidence douloureuse si efficaces: l'honneur du marquis de Croixmare en qui l'héroïne met sa confiance, à qui elle livre ses secrets les plus dangereux, se trouve invoqué.

D'un texte à l'autre on peut donc suivre un cheminement. En 1721, les Persanes captives sont soumises à une contrainte stérile exercée par les eunuques impuissants mais violents pour le compte d'un maître absent, figure d'un Dieu ironiquement à la fois de plus en plus 'sage' alors qu'il découvre les ressources de la liberté, de plus en plus 'caché' puisqu'il est inaccessible à ses 'fidèles,' et de plus en plus jaloux alors qu'il ne peut accorder ses faveurs (ou sa Grâce?) et doit se borner à sévir de loin (comme Jupiter tonnant, lettre CLIV: 'puisse cette lettre être comme la foudre'). ${ }^{15}$ Elles ne peuvent affirmer leur liberté que par la désobéissance ou le désespoir suicidaire: le défi de Roxanne 'oui je t'ai trompé' (incipit de la lettre CLXI) n'est que l'affirmation d'un refus, une négation impénitente et suprême de l'autorité despotique. En 1740, Théophé, tirée du sérail, intériorise une doctrine janséniste ('je lui avois donné les Essais de Nicole ... La Logique de Port Royal était un autre livre que j'avois cru propre à lui former le jugement,' 175) pour marquer son autonomie de façon plus douce, mais tout aussi ferme. Mais elle est en présence d'une figure d'autorité civilisée. Le Narrateur est homme d'honneur et généreux; il pardonne les offenses; il fait en général confiance à Théophé (malgré les machinations des conseillères, chaperons, compagnes ou duègnes de celle-ci, corrompues par les habitudes de l'orient ou le goût du pouvoir subalterne, de Bema (129) à Madame de xxx (271) en passant par Maria Rezati (193) - et qui toutes le haïssent plus ou moins ouvertement); ${ }^{16}$ il n'abuse pas de son pouvoir sauf à la fin, lorsqu'il manifeste sa jalousie pessimiste en imposant le silence. La difficulté de la relation entre l'Ambassadeur (véritable représentant de l'homme de qualité français face à des orientaux, fiers despotes/métèques trop souples/ou esclaves) tient cependant à l'incertitude où se trouve sa 'pupille' à son égard. Elle s'adresse d'abord à lui sur le mode de l'adoration, sous les noms de 'son Libérateur, de son Père, et de son Dieu'; ${ }^{17}$ elle déclare n'avoir d'obligation 'qu'à sa générosité,' et considère son 'amitié' comme un bien 'si précieux qu'il lui tiendra lieu éternellement de fortune et de plaisir' (169), autrement dit, laïcise cette 'soumission naturelle,' et la socialise en fonction des bienséances, car Son Excellence est un personnage influent; mais en même temps elle sait qu'il est un homme privé (o combien!) parfois tendre, parfois même pressant mais aussi jaloux, tâtillon, 
voire ridicule (tout à la fin, la scène où il se baisse pour 'observer le dessous du lit,' 266) hésite entre le comique et le pathétique, et de son propre aveu, il se trouve parfois dans une situation proche de celle des barbons de Molière (247): quand le jeune de S... lui confie ses projets d'enlèvement on pense à Horace en présence d'Arnolphe. Il est difficile à la jeune femme de se trouver de plain-pied avec lui.

En 1760, chez Diderot, Dieu est bien Dieu pour Suzane, et le protecteur n'est pas un personnage du roman. La mystification du 'charmant Marquis' est certes inscrite dans les rappels phatiques, et, nous l'avons vu, sous-tend les stratégies de la caution, du leurre, et de la justification: mais $M$. de Croixmare n'est pas présent dans le texte (quant à Dieu, il n'est pas l'objet d'une transposition idolâtre, même si les gens de l'appareil abusent de son nom et si l'ex-religieuse, croyante sincère, invoque encore son jugement).

D'autre part, la parole féminine est aussi beaucoup plus soutenue et riche. Il n'est peut-être pas sans pertinence de noter qu'elle semble avoir pour horizon un idéal d'indépendance plébéienne, une certaine aspiration à une vie dépouillée mais digne: 'de la sécurité, du repos d'esprit s'entend du pain et de l'eau.' Cette vie ressemble à celle du cloître (de même que celle du cloître ressemble à celle du sérail pour Théophé ('Je pense... à me faire un ordre de vie tel que je l'observais à Oru, et de tous les lieux dont j'ai pris ici connaissance, je n'en vois point qui soit plus conforme à mes inclinations qu'un couvent' disait celle-ci, H.G.M., 260). Pourtant la liberté s'y définit de façon intérieure, et non pas en fonction d'une règle impossée, ou de voeux perpétuels (à cet égard, le couvent, où l'on entre en principe volontairement Suzanne rappelle cet engagement - pour l'abolir - à deux reprises: à propos de la première profession arrangée: 'on était résolu à disposer de moi sans moi,' 96) qu'elle ne peut enrayer que par un scandale:

Est ce de votre plein gré et de votre libre volonté que vous êtes ici? Je répondis, non, mais celles qui m'accompagnaient répondirent pour moi, oui. (100)

et lors de sa prise d'habit effective ('on disposa de moi pendant toute cette matinée qui a été nulle dans ma vie, car je n'en ai jamais connu la durée' [123] - est cependant plus 'libre' que le sérail où vivent les esclaves).

L'éventail des options s'ouvre donc entre le sort des femmes esclaves, celui des femmes 'libres,' mais dépendantes, celui des travailleuses autonomes, qui ne souhaitent 'qu'une condition supportable s'il se peut, ou une condition telle quelle' (La Religieuse, 287). Il se trouve qu'il correspond à trois régimes politiques: le fameux 'despotisme oriental' 
(fondé sur l'intimidation d'esclaves), la société aristocratique française d'Ancien Régime, avec ses ordres privilégiés mais tenus par des obligations, et qui repose sur l'honneur, la société démocratique qui s'ébauche, et dont les membres aspirent à l'égalité dans le travail, sans savoir encore si ces valeurs et ces atouts du Tiers État définiront une République bourgeoise ou un régime spartiate: c'est là que les femmes, locutrices manifestes, permettent de mettre en scène les préoccupations des écrivains qui se cachent pour réfléchir à l'histoire et à l'esprit des lois humaines et en parler; l'éventail sert alors de masque. Mais les paroles assignées (expression positive des lumières et des ombres) débouchent aussi sur le silence, remontée vers l'en-deçà des lois et des institutions, vers le lieu mythique de la femme 'non-née' ou 'nonavenue,' absence à la prise institutionnelle, et évanouissement, ou plongée vertigineuse vers l'au-delà du pensable. Et à travers ces 'blancs' de l'Histoire où l'éventail s'arrête (par pudeur? par angoisse?) ${ }^{18}$ passent la spéculation mythique et l'échange spéculaire avec l'Autre.

Paroles assignées aux femmes, relation qu'en donnent les personnages masculins qui s'arrogent le titre de porte-paroles, discours des pouvoirs visant à circonscrire ces paroles sont présents dans nos trois romans. Toutefois les discours l'emportent chez Montesquieu, qu'ils se présentent comme l'expression didactique et/ou exhortatoire d'une doxa (lettre XXVI, d'Usbek à Roxane) ou d'une réflexion dialectique (la fameuse 'grande question' de Rica, dans la lettre XXXVIII 's'il est plus avantageux d'ôter aux femmes la liberté que de la leur laisser,' qui, on le remarquera se pose 'parmi les hommes,' à l'exclusion des intéressées), et les relations-témoignages chez Prévost (avec atténuation corrélative des discours qui témoigne de l'incertitude du narrateur et qui n'est frappant, aux yeux de Théophé - par un mouvement de 'remontée vers l'autorité' - que parce qu'il n'avait aucune place au sérail, lieu des contraintes et des intrigues: 'l'ordre d'un discours sensé que je vous entendis prononcer me rendit d'abord extrêmement attentive,' 40). ${ }^{19}$ Chez Diderot le schème s'inverse, puisque (dans l'univers du texte romanesque), c'est une femme qui rapporte les discours des autorités - hommes, et femmes détentrices d'une autorité locale déléguée, analogues des eunuques des Lettres Persanes - et qui, en outre, place ces discours en porte-à-faux et en révèle le vide en les confrontant, par une habileté spontanée, avec une parole pleine. ${ }^{20}$

D'autre part, Montesquieu, puisqu'il donne (même brièvement) la parole à plusieurs femmes, distribue entre des caractères distincts les modalités de résistance au pouvoir.

Certes les trois 'meneuses' du sérail sont toutes les trois durement réprimées à la fin du roman ('nous n'avons plus rien de libre que les pleurs' écrit Roxanne dans la lettre $C L V I$ ). Mais leurs réactions sont 
alors distinctes, allant de la protestation pathétique (Zachi, lettre CLVII), à un détachement stö̈que (le très court billet de Zelis, lettre CLVIII), à un défi suicidaire (Roxanne). Or, dès le début du roman Zachi définissait 'sa liberté à travers un jeu de bienséances et une dialectique du divertissement' ('nous partîmes pour la campagne où nous espérions être plus libres' [XLVII]; l'évanouissement même semblait alors être 'tactique'). Roxanne, par contraste conservait une volonté farouche $\mathrm{d}^{\prime}$ 'indépendance là même où l'institution semblait la livrer totalement, défendant sa chasteté contre Usbek, son maître et époux 'par les refus obstinés d'une pudeur alarmée' (lettre XXVI) et allant jusqu'à le menacer d'un poignard. Au sein même de l'esclavage donc, Zachi a opté pour le compromis (et à la fin elle en appelle encore à Usbek dont elle espère le retour: c'est un personnage de comédie larmoyante) alors que l'entière Roxanne affirme des valeurs absolues: 'J'ai pu vivre dans la servitude mais j'ai toujours été libre: j'ai réformé tes lois sur celles de la Nature, et mon esprit s'est toujours tenu dans l'indépendance' (lettre CLXI).

Prévost, et surtout Diderot procèdent autrement: c'est chez le même personnage ('scripteur') féminin que se retrouvent accommodements et ruses, conduites d'évasion et de repli, et affirmations ouvertes de l'indépendance, mais selon plusieurs étapes. Théophé, qui connaît une conversion lorsque le narrateur la libère, qui adopte un nom nouveau pour remplacer celui de Zara 'qu'elle portait dans l'esclavage' et qui va parcourir encore plusieurs cycles où elle passera de la tentation au rachat mais toujours de façon relative, puisque nous n'avons jamais de preuves de sa chute ni d'assurance de son salut (ce qui explique la cruelle incertitude de l'Ambassadeur et les affres de sa jalousie), a commencé par accepter tranquillement la dégradation: 'Je suis moins tombée dans le désordre que je n'y suis née. Aussi n'en ai-je jamais connu la honte ni les remords (25).' À travers les aventures romanesques et sanglantes qu'elle rapporte, elle conserve une belle équanimité, assurée qu'elle est de toujours dépendre des hommes, mais aussi 'par [ses] complaisances' de pouvoir 'acquérir sur eux une espèce d'empire qui les mettoit à leur tour dans sa dépendance' (31). C'est pourquoi elle n'a pas hésité, dans le besoin, à se mettre elle-même en vente comme esclave (35-36). Qu'elle ait été trompée ou se soit trompée ne change rien à l'affaire. Que le désenchantement (au sens de Loti) ait succédé à la satisfaction fait d'elle une âme romanesque ('plus grande que le monde,' dirait Lukacs) et prépare le 'saut' de sa conversion. Mais comment peut-on être Française? Une fois 'libre,' elle intériorise les contraintes, les renforce de scrupules, les stoïcise et les teinte d'humanisme dévot, sans pour autant changer de 'caractère'; elle demeure une Grecque sans patrie. On pourrait voir dans son histoire 
une transposition de celle de Prévost, l'apostat repenti, incertain de son statut: à la question que prête Jean Sgard à l'abbé, 'ai-je été un faux moine?'21 répondrait alors celle de la 'convertie': 'ai-je trahi ma liberté?' Dans les deux cas on ne peut que souffrir en tentant d'expier un acte de mauvaise foi. L'acte même d'accéder à la liberté est pour Théophé une grâce qui divinise celui qui l'a rachetée, et tout commerce 'humain' avec lui serait sacrilège, et cause de perdition, pour elle, et pour lui. C'est pourquoi elle aspire au cloître car elle y trouverait la liberté de 'la femme de qualité qui se serait retirée du monde' - mais cette aspiration demeure conditionnelle et le narrateur jaloux lui refuse de se définir ainsi en tant que sujet.

Le cas de Suzanne est différent puisque les voeux qu'elle prononce 'librement' lui ont été arrachés, soit par la contrainte, soit par la duperie (le chemin de fleurs dont la mère des Novices couvre la voie qui conduit à la règle asservissante), soit par un appel à une 'raison' qui n'est que résignation. Elle est en quelque sorte appelée à expier un péché originel au second degré, celui de tous les humains déchus, dans l'univers chrétien, et la faiblesse particulière de sa mère adultère: si le baptême l'a rachetée du premier, il faut un autre sacrifice pour compenser le second (il est donc particulièrement ironique que Mme Simonin puisse s'écrier: 'Dieu nous a conservées l'une à l'autre pour que la mère expiât sa faute par l'enfant' [109] puisqu'elle dispose abusivement d'une âme, et étend au spirituel et au privé les sacrifices de leur progéniture qu'acceptent et imposent Agamemnon ou Abraham). ${ }^{22}$

Cependant, elle aussi, la Religieuse vertueuse et innocente, qui a toute notre sympathie, est capable de ruses et de mensonges, tout excusés qu'ils soient par une légitime défense. Mais, à la différence de ses soeurs 'orientales,' elle dialectise son opposition (et ce même lorsqu'elle est soumise aux pressions les plus intenses, privées - comme lorsqu'elle défend son recours juridique ['protestation contre ses voeux'] devant la supérieure, 152-57 - ou publiques - lorsqu'elle comparaît devant l'archidiacre 'au moment le plus terrible de sa vie,' 162, 174-78), ${ }^{23}$ elle l'articule à partir de lois positives et d'arguments rationnels qui, bien qu'établis par l'appareil même qu'elle combat, définissent les conditions mêmes de sa lutte ('actions que vous appellerez ou imprudence ou fermeté,' 129-30). Son appel au Marquis est sous-tendu par une série de procédures et de plaidoyers, proches des Lettres, des Mémoires, des Réfutations et de Apologies qui forment une partie si importante des Oeuvres philosophiques de Diderot, et animé par des Dialogues vigoureux.

Mais les personnages féminins des trois romans débordent aussi le 'dicible,' vers l'en-deçà de l'être, et vers l'au-delà de la conscience. Au siècle de Condillac, l'hypothèse de l'éveil de la statue n'est pas seulement un moyen commode d'exposer (par un 'gradus') les thèses 
sensualistes; elle se nourrit de souvenirs mythiques (Jean Fabre note, à propos de H.G.M., 'une fois de plus, la statue s'anime contre Pygmalion') qui peuvent déboucher sur une évocation de la création (êtres pétris d'argile) et de la naissance miraculeuse de la conscience (qui pourtant précède la chute mais indiquera plus tard les chemins de la liberté): c'est précisément le point d'équilibre troublant entre la création (en tant qu'objet) et la naissance (en tant que sujet) qui préoccupe les penseurs des Lumières en général (on pourrait citer Fénelon, déjà, et Voltaire, et Marivaux, et Condorcet, et jusqu'à Sade - sans parler d'auteurs moins connus, ni bien entendu de Rousseau!) et nos trois romanciers en particulier. Le même point d'équilibre se retrouve lorsque l'on perd la conscience. J'ai parlé de l'évanouissement 'tactique' de Zachi par exemple. Mais il peut être un blanc, une absence profonde, pour Suzanne 'physiquement aliénée' (124) lors de sa profession (et qui entre machinalement, par un rituel auquel sa conscience se dérobe, dans la 'vie' du cloître comme ceux qui reçoivent les sacrements sont préparés [sans s'en rendre compte vraiment] à entrer dans la mort); à la fin de son histoire elle est suspendue dans ses souvenirs (à l'indignation de la femme pratique qui l'a recueillie ['à quoi rêvez-vous donc!']; tout au long du récit elle est 'absorbée,' 'stupide' - elle ordinairement si raisonnable, si diserte, si habile dialecticienne - aux moments de grande crise; elle agit ainsi sans être responsable (avec l'immunité du fou, mais temporaire, et bienséante). D'autre part cependant la servitude même, en réifiant les femmes, leur confère une 'innocence' ambiguë, de par sa passivité: celle dont Usbek félicite Roxane (lettre XXVI: 'Que vous êtes heureuse! Vous vivez dans mon sérail comme dans le séjour de l'innocence... vous vous trouvez avec joie dans une heureuse impuissance de faillir'), celle dont la mère des Novices berce ses pupilles (91), 'qui vous endort, qui vous en impose, qui vous fascine,' et surtout celle que le Narrateur de l'H.G.M. tient à attribuer à Théophé, alors même que celle-ci a quitté le sérail, en venant même à lui attribuer une espèce de singularité 'Evo-mariale' quand il compare sa situation à celle de la Sicilienne Maria Rezati, laquelle s'est 'attiré ses infortunes par une faute volontaire':

Peut-être êtes-vous l'unique exemple d'un malheur innocent dans le même genre, et la seule personne de votre sexe qui après avoir été entraînée dans le précipice sans le connoître ait changé $d^{\prime}$ inclination, au nom et à la première idée de la vertu. (159)

Tout se passe comme si, par un privilège unique, Théophé était à la fois 'statue' et femme, objet (incapable de connaissance) et vierge de tout soupçon, de toute souillure, immunisé contre la chute, mais en 
même temps sujet, actif et libre, puisqu'elle a changé d'inclination, et ce, par un appel de la grâce. On conçoit que cet être impossible à concevoir soit impossible à comprendre!

Pour les hommes, désespérément sujets, le refuge, le retour à 'l'innocence' sont impossibles (le statut des Eunuques puissants/impuissants suppose une mutilation traumatisante, 'une exécution mille fois plus cruelle que la mort' comme dit l'esclave mâle Pharan [Lettre Persane XLII] que l'on veut castrer pour le 'distinguer'). ${ }^{24}$

L'éventail des figures féminines se retourne alors et la femme est dominatrice (comme le croient les pensionnaires du sérail d'Usbek et Théophé elle-même, avant et peut-être après sa 'conversion': Fabre évoque La Femme et le Pantin, on pourrait pousser jusqu'au Professor Unrat de Heinrich Mann qui a inspiré 'L'Ange Bleu'). Mais cette liberté illusoire de l'automate/autonome est au fond monstrueuse: on sait ce que l'imaginaire 'noir' en fera à la fin du siècle des Lumières, avec le fiasco de Frankenstein ou de Coppelius. Certes Diderot (sous le masque de Julie de Lespinasse précisément) avait déjà envisagé que 'l'homme n'est peut-être que le monstre de la femme et la femme le monstre de l'homme,' mais en termes de 'limite' physiologique. ${ }^{25}$ On peut se demander si, inconsciemment nos trois romanciers n'avaient pas perçu le problème en termes 'théologiques' et mythiques. Le dit de la femme serait alors le non-dit de l'homme mais le non-dit de la femme attendrait l'étincelle néantisante de la liberté pour tous les humains, comme le retrait scandaleux de Suzanne désespérée - à travers de atrocités qui ouvrent la perspective de la damnation (178) - ou le suspens qui arrête sa plume alors qu'elle en appelle au salut même de son destinataire masculin (287) peuvent le laisser spéculer.

\section{PIERRE GOBIN}

Queen's University

\section{Notes}

1 La violence sacrilège des Eleuthéromanes de Diderot ne peut s'exercer que dans le privé.

2 Diderot encore, dans la célèbre lettre à la Princesse Dashkoff du 3 avril 1771 (ed. Roth, vol. XI, pp. 17-21. La citation souvent reprise 'chaque siècle a son esprit qui le caractérise. L'esprit du nôtre semble être celui de la liberté' est à la p. 20 et concerne ' 1 'affaire' La Chalotais et le conflit des Parlements). 
3 C'est Thomas Kavanagh qui emploie cette image du miroir vide ('The Vacant Mirror, A Study of Mimesis Through Jacques le Fataliste,' S.V.E.C., 104 [1973]).

4 Cf mon article 'L'Arlequin-Deucalion de Piron: pertinence de l'impertinence,' S.V.E.C. 192 (1980), 1478-486.

5 C'est peut être pourquoi les femmes 'savantes' (Mme du Chatelet) ou trop 'sérieuses' (Mme de Lambert, Mme de Graffigny, Mme Necker) ne réussirent pas vraiment, en tant qu'hôtesses, à établir une 'cour.' La 'Reine des Lanturelus' elle-même, la Marquise de la Ferté-Imbault (née Geoffrin) suscita les soupçons quand elle voulut monter une entreprise contre-philosophique. Cf Constantin Photiades, La Reine des Lanturelus (Paris: Plon, 1928), notamment pp. 154-55.

6 C'est un des éléments qui 'inspirèrent' la Métromanie. Diderot, dans le 'PostScriptum' de la Religieuse montre un mouvement de balancier entre la confiance et la méfiance qui résultent de ce charme, lorsqu'il introduit les soupçons de la fictive et vertueuse Suzanne: 'Cependant, si le marquis, à qui l'on accorde le tact le plus délicat, venait à se persuader que ce n'est pas à sa bienfaisance mais à son vice que je m'adresse, que penserait-il de moi? Cette réflexion m'inquiète.' Mais aussi il désarme les clichés sexistes (p. 286 de l'édition critique publiée en 1975 chez Hermann), à la fois en les refoulant dans une hypothétique 'nature' féminine qui échappe à la conscience analytique du sujet tout en l'éveillant (un 'que sais-je' qui travaille le 'je ne sais quoi' ineffable qui n'est pas nécessairement inexistant, comme l'a rappelé Jankélévitch), et en les mettant au service des jeux entre 'la' vérité nue et ses 'vêtements,' ou entre la 'nature' et l'artifice: 'En vérité, il aurait bien tort de m'imputer personnellement un instinct propre à tout mon sexe. Je suis une femme, peut-être un peu coquette, que sais-je? Mais c'est naturellement et sans artifice.

7 En 1926, F.C. Green avait 'instruit' le dossier; Deloffre et Rougeot ont pu rendre un verdict décisif dans leur édition de 1962. Guilleragues, ambassadeur en Turquie, n'est d'ailleurs pas sans évoquer le personnage du narrateur de l'Histoire d'une Grecque Moderne.

8 Cf. mon travail sur 'le dénouement de Tartuffe et le spectateur privilégié,' Actes du VIle congrès de l'AILC/ICLA (Budapest: Akademiai Kiado, 1976), 281-86.

9 Dans la perspective proposée par Greimas. L'analyse du secret dans son Maupassant (1976) recoupe la perspective qui nous intéresse ici.

10 J'ai concentré mon attention sur la période $1720-1760$, et les textes qui marquent la tension entre les contraintes de la claustration et les aspirations à la liberté personnelle: le lamento amoureux des Lettres de la Religieuse Portugaise (1699) 'prolongement de la tragédie racinienne' et le plaidoyer idéologique ouvert des Victimes cloitrées de Boutet de Monvel (1791), sont donc exclus.

11 Ce sont les 'kapos' infernaux de cet univers concentrationnaire. Il y a toute une étude à faire sur l'orgueil de ces déchus/amputés.

12 Je renvoie sur ce point à l'excellente petite présentation de Vivienne Mylne (Diderot's La Religieuse, 1981) ainsi qu'à l'article où Jacques Proust établit des rapprochements précis entre les doléances de Suzanne et des 'cas' d'abus rapportés par les très jansénistes Nouvelles Ecclésiastiques ('Recherches nouvelles sur La Religieuse,' Diderot Studies, 6 [1964], 197-214). 
13 En effet l'Amant d'une belle devrait avoir reçu des gages manifestes de la réciprocité des sentiments qu'il avoue. Or le reste de l'histoire nous révèle que si Théophé a voué au narrateur l'affection et le respect dûs à un père ou un maître (ou même à la limite une espèce d'adoration le 'divinisant' [164]) jamais elle ne l'a accepté comme partenaire mâle, en tant que maîtresse ou épouse, en dépit de ses avances. Dès la première page la narrateur se corrige subtilement: 'J'ai longtemps aimé, je le confesse encore... Mais l'amour n'a jamais eu pour moi que des rigueurs... Je suis un Amant rebuté, trahi même si je dois m'en fier à des apparences dont j'abandonnerai le jugement à mes Lecteurs. Estimé néanmoins de ce que j'aimois, écouté comme un Père, respecté comme un Maître, consulté comme un Ami; mais quel prix pour des sentimens tels que les miens!' (3-4).

14 (P. 274 de l'édition Hermann, cf. appendice de la même éd. p. 294.) Cette métaphore du 'déchirement du voile' fonctionne à plusieurs niveaux: primaire (le secret se révèle, et la nature du péché de la supérieure) 'secondaire' et structural (la reconnaissance de l'abîme qui s'ouvre devant Suzanne - et même devant toute religieuse avant la 'sagesse forcée' qu'est censée apporter la ménopause cf. 'Il est sûr monsieur que sur cent religieuses qui meurent avant cinquante ans il y en a cent tout juste de damnées, sans compter celles qui deviennent folles, stupides ou furieuses en attendant' (p. 91 éd. Hermann) rend nécessaire une action violente: on ne peut espérer alléger ou réarranger le voile en changeant d'ordre par exemple, ou de supérieure; il faut à tout prix quitter le couvent, arracher le voile que l'on a dû prendre malgré soi; la situation présente de Suzanne fugitive, marquée à la fois par sa rupture extérieure de clôture et la rupture mentale qu'elle doit faire avec son éducation et ses habitudes pour se perdre dans le Tiers Ètat après avoir été 'couverte' par l'ordre du clergé s'explique alors. Pour une nonne, déchirer le voile, c'est l'équivalent de défroquer pour un moine), et peut être même au niveau du non-dit sexuel (Suzanne, 'innocente' jusqu'alors est en quelque sort violée, non par le regard de vieillards libidineux - ce n'est sans doute pas par hasard qu'elle s'appelle Suzanne - mais par la parole d'une femme mûre et dévergondée. Le voile c'est l'hymen de la pucelle. L'héroïne demeure certes vierge physiquement; mais mentalement elle n'est plus la Chaste Suzanne, elle vient d'être 'déflorée').

15 Usbek est cependant donné pour 'bon' et il est, au départ, chéri de ses créatures qui, elles, sont pleinement révélées à ses regards (lettre III, de Zachi:... 'que de charmes furent étalés à tes yeux... tu portas tes curieux regards dans les lieux les plus secrets...'). Mais en tant que Dieu/Maître présent il peut alors répondre à la 'passion' de sa zélatrice en lui accordant une grâce personnelle. Après son départ, il conserve son prestige en dépit de son absence et on lui rend encore un culte exact: 'Quoique je ne doive être vue de personne et que les ornements dont je me pare soient inutiles à ton bonheur, je cherche cependant à m'entretenir dans l'habitude de plaire' (Fatmé Lettre VII). Mais les choses se gâteront lorsque les femmes, tout en trichant avec la contrainte, oseront se vanter ' $d$ 'une vertu qui n'est pas libre' (Lettre XX, Usbek à Bachi, sa femme... on notera cependant que le rapport demeure singulier avec la fidèle/infidèle). Rica cependant découvrira un 'grand exemple de tendresse conjugale chez une occidentale (libre et même reine) et associera cette grandeur à la philosophie (CXXXIX). L'apologue en abyme de la Lettre CXLI, même s'il finit bien puisqu'un 'céleste Ibrahim' se substituant au 
jaloux absent libère le sérail, se montre libéral avec les biens du jaloux avare qu'il 'dissipe avec une immense profusion,' et répand généreusement sa fécondité puisqu'il trouve le moyen de faire 'trente-six enfants' à ses femmes, laisse prévoir les désordres qui vont précipiter la catastrophe (à partir de la Lettre CXLVII du grand Eunuque).

16 On peut opposer cette haine envers le narrateur des femmes domestiques au dévouement des serviteurs français mâles qui ne se dément pour ainsi dire jamais dans H.G.M. (alors que les Grecs y font souvent preuve de duplicité) Stéréotypes sexistes? Xénophobie? Clichés culturels? Application de théories des climats et des gouvernements voisines de celles de Montesquieu? La question mérite une étude.

17 Le Chevalier de Malte en rupture de voeux et qui évoque, et Prévost, et Des Grieux introduira une note néo-testamentaire dans cette relation lorsqu'il remerciera Le Narrateur de l'avoir tiré de l'esclavage: 'la grâce qu'il me demanda à genoux en me donnant le nom de son Père et son Sauveur [NB: Les Capitales sont dans le texte!] fut de permettre qu'il prît aussi-tôt un autre habit' (164).

18 Les vers d'éventails de Mallarmé privilégient la 'proscription.' 'l'effacement,' 'L'aile du Temps (qui se) referme.' C'est une autre façon pour le scripteur masculin d'appeler la femme (destinataire ici) à ' $\left[\mathrm{l}^{\prime}\right]$ introduire dans [ $\mathrm{s}$ ']on histoire' - texte utilisé précisément par Proust dans Albertine disparue. Le 'souffle' de l'éventail (brise), brise aussi la statue en la constituant dans le silence et par le regard 'orthogonal' et direct:

Autour du marbre, le lys croît -

Brise, ne commence par taire

Fière et blanche son regard droit

Nelly pareille à ce parterre.

(Éventail VI [ed. Pléiade], 108)

À l'exception du dernier vers 'dédicatoire,' ce quatrain articule la problématique de l'éventail sur celle de la statue - ô profondeur du 'frivole' imputé à Condillac par Derrida!

19 On se souviendra que dans ce discours l'Ambassadeur posait précisément la même question que Rica, mais sur le mode pathétique et non plus en termes enjoués (amorce d'Un Armour de Swann, comme l'ont noté Jean Fabre

['L'Abbé Prévost et la tradition du roman noir' in L'Abbé Prévost (actes du colloque d'Aix en Provence, Annales de la Faculté des Lettres d'Aix, 1965), 54], et Francis Pruner ['Psychologie de la Grecque Moderne,' 140-46 du même recueil, et notamment p. 144], plutôt que de La Ronde de Schnitzler). Surtout il envisageait ce problème brûlant en s'adressant à une femme: même si c'est pour parler 'avec douleur de l'infortune des Pays Chrétiens,' il évoque le statut dont y jouissent ses semblables, et ironiquement, croyant la flatter (comme Usbek s'adressant à Roxanne), introduit dans son esprit un ferment d'insatisfaction.

20 Vivienne Mylne (op. cit. p. 61) a bien montré comment, lorsque Suzanne refuse publiquement de prononcer ses voeux, sa prise de parole insolite et déconcertante met en évidence l'artifice et la manipulation du discours de l'appareil: "The scandal arises from Suzanne treating... the "ritual queries" (purely formal since the answers are known in advance) as real questions.' 
21 'L'apostasie et la réhabilitation de Prévost' in L'Abbé Prévost, op. cit., 17.

22 Les abus dont est victime Théophé sont différents, puisqu'ils sont le fait de pères supposé et/ou incertain.

23 Bien que Diderot n'ait pu évidemment connaître les réponses de Jeanne $\mathrm{d}^{\prime}$ Arc à ses juges (la publication par Quicherat du procès date de 1840 environ), Suzanne a la même attitude de bon sens et de simplicité accusatrice, qui transcende les procédures inquisitoriales de la justice de l'appareil et emporte l'adhésion par une espèce de confiance en la Justice absolue. C'est un admirable tour de force de la part de 'l'athée' Diderot!

24 Op. cit., 53.

25 Le Rêve de d'Alembert (Oeuvres Philosophiques, éd. Garnier), 328. 